\title{
Interaction Mesh Based Motion Adaptation for Biped Humanoid Robots
}

\author{
Shin'ichiro Nakaoka ${ }^{12}$ and Taku Komura ${ }^{1}$
}

\begin{abstract}
Adapting human motion data for humanoid robots can be an efficient way to let them conduct specific tasks or perform visually intriguing movements. However adapting movements which involve close interactions between body parts / objects has been a difficult problem if we try to make the motions sufficiently similar to the original ones without causing undesired collisions and fall-overs. To solve this problem, we propose a novel motion adaptation system which can adapt full-body motions of a human to those by a biped humanoid robot. The system integrates the joint coordinate model and a dynamic balancing method with the interaction mesh based motion adaptation method, which is originally designed for character motions represented by marker positions in Cartesian coordinate. We present that the system can adapt human motion sequences that include close interactions between the arms for the biped humanoid robot $\mathrm{HRP}-4 \mathrm{C}$, and the adapted motion can be stably performed in dynamics simulation.
\end{abstract}

\section{INTRODUCTION}

In many cases, humanoid robots are expected not only to visually appear like humans but also to conduct natural looking movements. However, synthesizing such movements using existing algorithms is not an easy task. Adapting human motions for the humanoid robots can be a reasonable solution for this problem. It can also make it easier to obtain complicated motions of a robot body with large degrees of freedom (DOF) as humans can easily move their bodies without difficulty. Adapting human motions will lead to practical applications including the entertainment content player [1], preservation of intangible cultural heritages [2], and tele-existence [3]. The goal of this study is to develop a motion adaptation system for biped-type humanoid robots that can handle close interactions between body parts, and those between a robot and the environment.

In fact there have been studies which have moved humanoid robots by adapting captured human motions. Pollard et al. [4] developed a method for converting joint angles considering joint angle ranges and joint angular velocity limits, and the humanoid robot DB imitated human dancing motions with their method. Since their method only considers the trajectory conversion in joint-space, it could not move the whole body of a biped-type robot while keeping its dynamic balance. Nakaoka et al. [2] developed a method which can handle whole body motions including leg steps.

\footnotetext{
${ }^{1}$ S.Nakaoka and T. Komura are with Institute of Perception, Action and Behaviour (IPAB), School of Informatics, The University of Edinburgh, 10 Crichton Street, Edinburgh, EH8 9AB, United Kingdom, tkomura at ed.ac.uk

${ }^{2} \mathrm{~S}$. Nakaoka is with Intelligent Systems Research Institute, National Institute of Advanced Industrial Scicence and Technology (AIST), Tsukuba Central 2, 1-1-1 Umezono, Tsukuba, Ibaraki 305-8568 Japan. S . nakaoka at aist.go.jp
}

They demonstrated the biped humanoid robot HRP-2 [5] can imitate the whole body motion of a human dancer. Yamane et al. [6] developed a method for controlling whole body motions of a biped humanoid robot to follow captured human motions. Although the way of foot stepping their method can handle is limited to quasi-static one and the experiment using an actual robot has only been done for motions which do not include steps, their method can achieve the on-line adaptation with a short delay.

In these studies, self-collision problem is not well considered. The motion adaptation is a process to convert the motion while taking into account the differences of the joint structures, sizes of body parts, and dynamic balance. If this process does not consider collisions, a motion that involves close interactions can easily result in an adapted movement which include undesired collisions unseen in the original motion.

For this problem, Dariush et al. [7] proposed an online motion adaptation that avoids self-collisions. They showed that the humanoid robot ASIMO could follow human upper body motions. Kanehiro et al. [8] also proposed a motion adaptation method which can avoid self-collisions. The problem of these methods is that a resulting motion tends to be unsmooth and unnatural when it has continuous close interactions because the adjustment for avoiding self-collisions is only applied per frame where the distance between adjacent body parts is less than a certain threshold. This kind of the adjustment cannot necessarily keep the context of the original motion, and it might be stuck in local minima.

In the field of character animation, Ho et al. [9] proposed a method of adaptation that preserves the spatial relationship between body parts as mush as possible. In this method, the spatial relationship between body parts is represented by relative vectors that connect the adjacent joint positions. By minimizing the difference of Laplacian coordinates of the mesh structure that is composed by these relative vectors, the adapted motion can be similar to the original motion in terms of the spatial relationship. The body parts which are close / far in the original motion also become close / far in the adapted motion, respectively. This means that if the original motion does not include self-collisions, the adapted one does not include collisions, too. In contrast to methods which only consider the neighbor distance at each local frame, the resulting motion will be as smooth and natural as the original one because the relationship between all the body parts are taken into account at once, and the motion is adapted by spacetime optimization.

Although this approach has a good potential, we will 


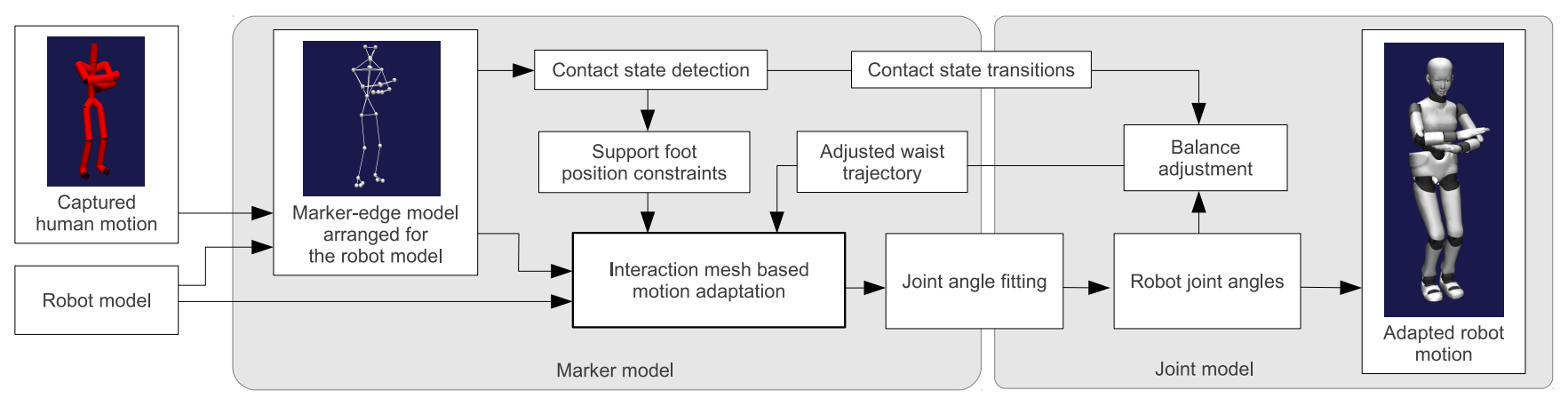

Fig. 1. Overview of the proposed system

face two problems when applying it for humanoid robots: Firstly, the method represents the character posture by joint positions, while humanoid robots use the joint angle representation. The joints usually have limited axes and range of rotations and the body parts cannot be brought to arbitrary positions in space. Secondly, in their method, the dynamic balance is not taken into account. Therefore, it is not guaranteed that the adapted motion can be stably performed by a biped humanoid robot, and the robot would fall down in most cases.

Our goal is to develop a method that can exploit the advantages of the spatial relationship preserving motion adaptation method for biped humanoid robots. We propose a system which integrates the joint-based model representation and the dynamic balance constraints to [9]. We tested the proposed system for the model of biped humanoid robot HRP-4C [10], and confirmed that the adapted motions can be stably performed in a dynamics simulation environment without self-collisions and fall-downs.

\section{Overview of the AdAPtation System}

Fig.1 shows the overview of our proposed motion adaptation system. In this figure, "Interaction mesh (IM) based motion adaptation" corresponds to [9], which is briefly described in section III. Other components are those we have newly developed and integrated with the IM component in order to handle biped humanoid robots. The key issues that we tackle in this extension are the followings:

1) The difference between the motion representation; the marker position and joint angle representation

2) Dynamic balance consistency between the body and the floor

First of all, the difference of the motion representations should be resolved. In the marker position-based representation, the joint positions can be anywhere as long as the bone lengths are preserved. However, a robot model cannot always follow such positions because its joint structures usually have less degrees of freedom and directions of offsets between joints may be different. To solve this problem, we introduce the "marker-edge model arrangement" and the "joint angle fitting" components, which are described in section IV.
Secondly, the original IM-based adaptation does not consider physical laws because it is not crucial for rendering the motions as animation. However, when we try to make a robot perform the motions, physical laws cannot be ignored, and especially the dynamic balance consistency between the robot body and the floor becomes critical for a biped-type robot to prevent falling down. Thus we need a method to keep the dynamic balance consistency and it must be integrated on the system. To solve this problem, we introduce the "contact state detection" and the "balance adjustment" components whose outputs are used as additional constraints in the IMbased adaptation. They are described in section $\mathrm{V}$.

By integrating all the above components, we achieved a system that adapts human motion to a given robot model. The output movements are as smooth as the original while there are no self-collisions nor fall-downs, even when the motion involves close spatial interactions between body parts. This is a novel achievement for the problem of adapting human motions to humanoid robots.

\section{Interaction Mesh BASED AdAPtATION Method}

Here we briefly describe the IM-based motion adaptation method [9]. In this method, poses are represented by marker positions as shown in Fig.2-(a). Markers are basically set to joint positions, and they can be also set to some other body parts. A pose of frame $i(1 \leq i \leq n)$ is represented by $\boldsymbol{V}_{i}=\left(\begin{array}{lll}\boldsymbol{p}_{1}^{i \mathrm{~T}} & \ldots & \boldsymbol{p}_{m}^{i \mathrm{~T}}\end{array}\right)^{\mathrm{T}}$ where $m$ is the number of markers, $\boldsymbol{p}_{j}^{i}$ is the position of $j$-th marker at frame $i$. Then adjacent markers are defined for each marker by applying the Delaunay tetrahedralizations [11] for each $\boldsymbol{V}_{i}$. The mesh structure connecting adjacent markers is called interaction mesh, which is shown in Fig.2-(b).

On the mesh, Laplacian coordinate [12] can be defined to represent the spatial relationship between markers. Laplacian coordinate of $\boldsymbol{p}_{j}^{i}$ is calculated by:

$$
L\left(\boldsymbol{p}_{j}^{i}\right)=\boldsymbol{p}_{j}^{i}-\sum_{l \in N_{j}} w_{l}^{j} \boldsymbol{p}_{l}^{i}
$$

where $L$ is the operator to compute the Laplacian coordinate, $N_{j}$ is the adjacent markers of $\boldsymbol{p}_{j}^{i}$, and $w_{l}^{j}$ are the normalized weights. The adaptation is processed based on the deforma- 


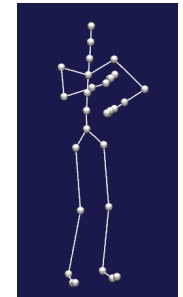

(a)

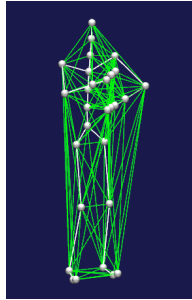

(b)

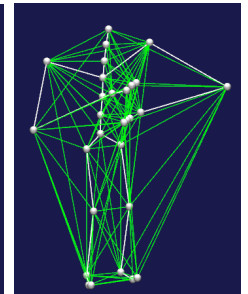

(c)

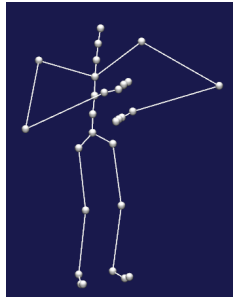

(d)
Fig. 2. Motion adaptation using the interaction mesh. (a) shows markers of the original motion. (b) shows an interaction mesh which is generated by applying Delaunay tetrahedralizations to the markers. (c) shows morphing to change bone lengths into those of the target model. The deformation energy defined on the interaction mesh is minimized so that the spatial relationship between body parts can be preserved as much as possible. (d) shows a pose of the final output, where the lengths of the arms are longer than the original ones.

tion energy which is defined as

$$
E_{L}\left(\boldsymbol{V}_{i}^{\prime}\right)=\sum_{j} \frac{1}{2}\left\|L\left(\boldsymbol{p}_{j}^{i}\right)-L\left(\boldsymbol{p}_{j}^{i^{\prime}}\right)\right\|^{2}
$$

where $\boldsymbol{V}_{i}^{\prime}=\left(\boldsymbol{p}_{1}^{i^{\prime T}} \ldots \boldsymbol{p}_{m}^{i^{\prime T}}\right)^{\mathrm{T}}$ is an updated pose. The adaptation tries to minimize the deformation energy, which means the spatial relationships between markers are preserved as much as possible.

During the adaptation, the bone-lengths must be changed into those of the target model. This is done by introducing the bone-length constraint

$$
\boldsymbol{B}_{i} \boldsymbol{V}_{i}^{\prime}=\boldsymbol{l}_{i} \text {. }
$$

where $\boldsymbol{B}_{i}$ is the Jacobian for the bone lengths, and $\boldsymbol{l}_{i}$ is a constant term vector.

Some marker positions can be constrained to be desired positions. This constraint is written as

$$
\boldsymbol{K}_{i} \boldsymbol{V}_{i}^{\prime}=\boldsymbol{P}_{i}
$$

where $\boldsymbol{P}_{i}$ is a vector representing desired positions.

In addition to the above terms, an acceleration energy term for reducing jaggy jumps between frames and a collision constraint term which considers the actual body part volumes for avoiding collisions between them are introduced.

The constraints are separated into soft ones $\boldsymbol{F}_{i} \boldsymbol{V}_{i}^{\prime}=\boldsymbol{f}_{i}$ and hard ones $\boldsymbol{H}_{i} \boldsymbol{V}_{i}^{\prime}=\boldsymbol{h}_{i}$, and the constraint energy for the soft constraints is defined as

$$
E_{c}\left(\boldsymbol{V}_{i}^{\prime}\right)=\frac{1}{2} \boldsymbol{V}_{i}^{\prime \mathrm{T}} \boldsymbol{F}_{i}^{\mathrm{T}} \boldsymbol{W} \boldsymbol{F}_{i} \boldsymbol{V}_{i}^{\prime}-\boldsymbol{f}_{i}^{\mathrm{T}} \boldsymbol{W} \boldsymbol{F}_{i}^{\mathrm{T}} \boldsymbol{V}_{i}^{\prime}+\frac{1}{2} \boldsymbol{f}_{i}^{\mathrm{T}} \boldsymbol{W} \boldsymbol{f}_{i}
$$

where $\boldsymbol{W}$ is a diagonal weight matrix.

The adaptation is done by iterative morphing. At every morph step, desired bone lengths and constraint positions are gradually changed towards the final target values, and the updated positions of all-frame markers $\mathcal{V}^{\prime}=\left(\boldsymbol{V}_{1}^{\prime \mathrm{T}} \ldots \boldsymbol{V}_{n}^{\prime \mathrm{T}}\right)^{\mathrm{T}}$ are calculated by solving

$$
\underset{\mathcal{V}^{\prime}, \boldsymbol{\lambda}}{\operatorname{argmin}} \sum_{i}^{n}\left\{E_{L}+w_{A} E_{A}+E_{C}+\boldsymbol{\lambda}_{i}^{\mathrm{T}}\left(\boldsymbol{H}_{i} \boldsymbol{V}_{i}^{\prime}-\boldsymbol{h}_{i}\right)\right\}
$$

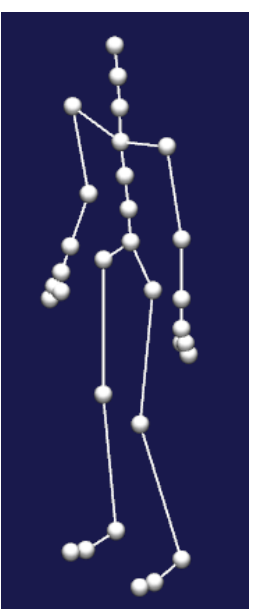

(a)

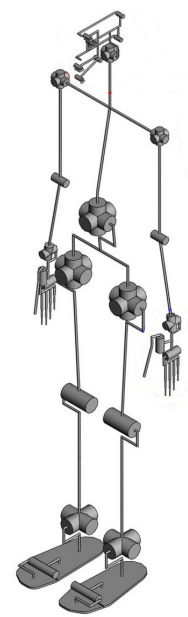

(b)

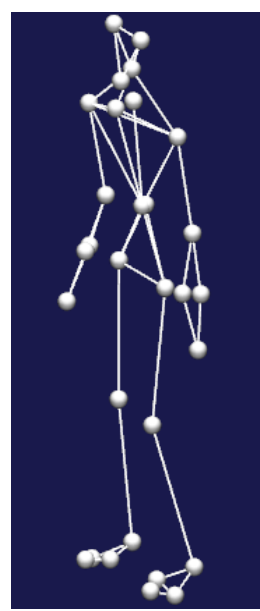

(c)
Fig. 3. Marker model arrangement considering a target robot model. (a) is a marker model directly derived from a motion capture skeleton. (b) is the joint model of HRP-4C. (c) is a marker model arranged for HRP-4C.

where $\boldsymbol{\lambda}=\left(\boldsymbol{\lambda}_{1}^{\mathrm{T}} \ldots \boldsymbol{\lambda}_{n}^{\mathrm{T}}\right)^{\mathrm{T}}$ is Lagrange multipliers, $E_{A}$ is the acceleration energy, and $w_{A}$ is a weight for the acceleration energy. This is a kind of quadratic optimization problem and the solution can be obtained by solving the linear equation derived from (6). Fig.2-(c) and (d) show an example of the adaptation, where the target model has longer arms.

\section{JOINT ANGLE MAPPING}

\section{A. Marker Model Arrangement}

Fig.3-(a) shows a typical marker model which can sufficiently express human body motions, and Fig.3-(b) shows an example of a robot joint model. The problem is that a robot model is usually more constrained than a marker model. In this example, the robot model lacks some joints which exist in the marker model. For example, the robot model does not have the joints of the spine and clavicles, which can be flexed in the original marker model. Even if there is a joint that corresponds to a marker, its movement could be more limited. For example, the markers of a hand can make an arbitrary orientation around the wrist marker, but a hand of HRP-4C can only rotate around the yaw and roll axes at the wrist joint because the joint only has the two axes. In this way, the number of joints and their DOFs of a robot model are usually less than those of a given marker model. In such a case, if we try to do the adaptation using the original marker model, the resulting motion would not match the robot model after all.

To solve this problem, we apply the following arrangements to the marker model used for the adaptation:

1) Removing extra markers

2) Adding bones to make some body segments rigid

3) Adding markers and bones to constrain DOF of a joint

4) Adding markers to adjust relative joint positions

First, extra markers are removed to make the actual DOF closer to that of the robot model. In the example of Fig.3, 
four markers along the spine are removed and joints along the spine are represented only by the waist and neck markers in the updated model (c). Although removing markers is a simple solution for getting a model closer to the robot model, it still leaves big differences in the model structures.

By adding extra bones whose lengths are constrained, some DOFs which do not exist in the target robot model can be fixed. For example, in the updated model (c), a bone between the hip joints and three bones which directly connect the shoulders and the waist are added. These triangle structures remove the DOFs inside the waist and chest segments. The hip joint positions are fixed to the waist segment, and the shoulder joint positions are fixed to the chest segment, which is same as the robot model. This is necessary to fit a set of joint positions which are attached to the same rigid body segment of the robot.

In some cases, adding a set of markers and bones around a joint can achieve the same DOF as the corresponding robot joint. In the arranged model (c), two markers and five edges are added around a wrist joint, and the original wrist marker and the edges connecting it are replaced with them. As a result, the wrist can only rotate around the yaw and roll axes, which is same as the robot model. Similar arrangement is also applied to the toe joints.

Adding a marker can be also used for adjusting relative positions of the joints attached to the same rigid body segment. In the HRP-4C model, the sagittal positions of the neck joint and shoulder joints are not exactly same as that of the waist joint. In fact they are attached at slightly backward positions. If this offset is not reflected in the adaptation, the head and chest segments of the robot always lean from the original attitude. To solve this problem, a maker is added at the collar position. The marker is gradually moved forward by the bone-length constraint while keeping spatial relationship with other markers. This conversely makes the neck and shoulder markers backward from the original positions, and the offset is introduced in the adaptation result.

\section{B. Joint Angle Fitting}

Even if we apply the arrangement for the marker model, there may still be some differences of the joint constraints, and the adaptation result cannot exactly match the robot model. For example, the HRP-4C model has an offset between an elbow joint and a wrist joint along the elbow joint axis, but it is difficult to reflect this offset by arranging markers. As a result, if the robot tries to make the same positions as the adapted ones for the elbow and wrist positions, the hand orientations cannot exactly match the adapted marker model. On the contrary, if the robot tries to make the same positions and orientations for the wrists and hands, the elbow positions cannot exactly match.

In a practical sense, this problem can be solved by minimizing the total errors of some key joint positions and orientations because the remaining differences of the models are not so large after applying the adaptation using the arranged marker model. In our implementation, for each frame, we first calculate the initial joint angles from the positional relationships between markers. Then, for each arm and leg, numerical inverse kinematics (IK) from the initial joint angles is applied to obtain the total error minimized solution. We use the damped least squares method [13][14] as follows:

$$
\Delta \boldsymbol{\theta}=\left(\boldsymbol{J}^{\mathrm{T}} \boldsymbol{W}_{e}^{2} \boldsymbol{J}+\lambda^{2} \boldsymbol{I}\right)^{-1} \boldsymbol{J}^{\mathrm{T}} \boldsymbol{W}_{e}^{2} \boldsymbol{e}
$$

where $\Delta \boldsymbol{\theta}$ is an update value of joint angles in the iteration of the numerical IK, $\boldsymbol{J}$ is a Jacobian Matrix, $\boldsymbol{W}_{e}$ is a diagonal weight matrix, $\lambda$ is a non-zero damping constant, and $e$ is an error from the adapted marker positions. For example, the joint angles of an arm are computed to minimize the following error value:

$$
\boldsymbol{e}=\left(\begin{array}{lll}
\Delta \boldsymbol{p}_{\text {elbow }}^{\mathrm{T}} & \Delta \boldsymbol{p}_{\text {wrist }}^{\mathrm{T}} & \boldsymbol{\omega}_{\text {hand }}^{\mathrm{T}}
\end{array}\right)^{\mathrm{T}}
$$

where $\Delta \boldsymbol{p}_{\text {elbow }}$ and $\Delta \boldsymbol{p}_{\text {wrist }}$ are the errors of the elbow and wrist positions respectively, and $\boldsymbol{\omega}_{\text {hand }}$ is the error of the hand orientation. Their priorities can be adjusted by setting $\boldsymbol{W}_{e}$. The joint angles of a leg are similarly calculated to minimize the knee and ankle positions and the foot orientation.

\section{Dynamic Balance Adjustment}

In order to make adapted motions dynamically stable for a target robot, we integrate the balance adjustment into the adaptation system. The balance adjustment is done based on the concept of ZMP [15]. Since ZMP is only defined when a robot is getting a floor reaction force and it assumes that a sufficient friction force can work to prevent a slip between a foot and the floor, the current system also assumes that input motions do not include movements such as jumping, running, and slipping.

\section{A. Detecting Contact States}

First of all we need to know how the contact states between a foot and the floor change in a given motion. When a foot is making a contact with the floor, it is in the support state. More precisely, the support state foot is getting a floor reaction force and supporting the weight of the body. When a foot is not in the support state, it is in the swing state. How these contact states of both feet change along the time-line needs to be known for the remaining processes.

To detect the contact state changes, our system uses a method proposed by Nakaoka et al. [2]. The method first detects swing foot phases for each foot by seeing the toe marker velocities. Then the remaining phases can be recognized as support foot phases, and the state change sequences of both feet can be obtained.

\section{B. Support Foot Position Constraint}

Even if the foot markers are adapted so that it can fit the foot joints of a robot, resulting foot positions in the robot model does not necessarily fit to the floor as shown in Fig.4(a). One reason for this is that humans often lift an ankle even when the foot is a support foot. In addition, there may 

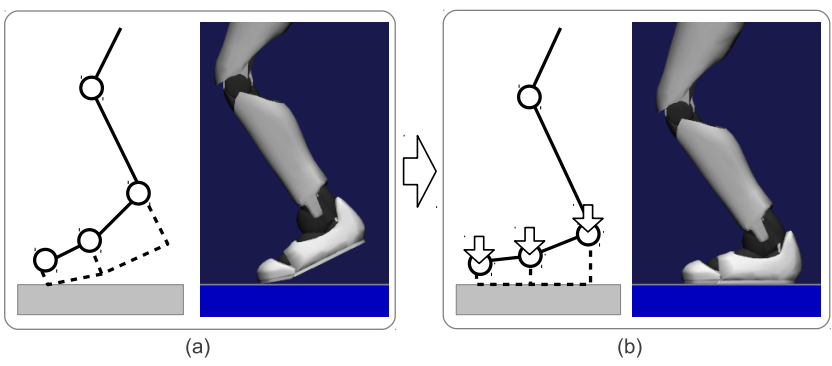

Fig. 4. Constraining a support foot position. Circles represent markers and dashed lines represent the foot surface. Original marker positions can be like (a), where the corresponding foot position in the robot model does not fit the floor. In (b), the vertical foot marker positions are constrained by hard positional constraints so that the heights can be same as the foot thickness, and the resulting foot position in the robot model fits to the floor.

be noises of the measurement in the motion capture system, or the marker may slightly swing on the foot. At any rate, in this case the foot cannot stably support the robot body and it would fall down.

To solve this problem, hard positional constraints are specified for the markers of a foot when it is in the support foot phase. As shown in Fig.4-(b), vertical positions of the foot markers are constrained so that the heights can be same as the foot thickness. In the morph steps of the adaptation, the desired heights are gradually changed from ones for the original human feet to ones for the robot feet. As a result, support foot positions corresponding to the adapted marker positions can firmly fit to the floor. This is a necessary condition for a robot to support its body stably.

\section{Calculating the Balanced Waist Trajectory}

In addition to fitting a support foot to the floor, an adapted robot motion must satisfy the dynamic consistency between its body and the floor so that it can follow the motion without falling down. Usually adapted motions obtained by the above process do not satisfy it because the dynamic consistency is not taken into account by the process in spite that the kinematic and dynamic properties are different between the original human body and the robot body.

To solve this problem, the system calculates the modified waist trajectory for the adapted motion so that the modified one can satisfy the dynamic consistency. Here we use a desired ZMP trajectory and a method which adjusts the waist trajectory so that the ZMP can be same as a given desired one.

A desired ZMP can be automatically determined from the foot contact state sequence. When a single foot is in the support foot phase, ZMP should be inside the sole of it, and when both feet are in the support phase, ZMP should be inside the convex hull consisting of the soles. In our system, the desired ZMP is set to the center of those areas to obtain the maximum stability margin.

Then our system uses a method proposed by Nishiwaki et al. [16] to adjust the waist trajectory according to a desired ZMP trajectory. In the original method, the relationship between ZMP and the center of mass $(\mathrm{CM})$ is written as

$$
\Delta p_{x i}=\frac{-z_{c} \Delta x_{i-1}+\left(2 z_{c}+g \Delta t^{2}\right) \Delta x_{i}-z_{c} \Delta x_{i+1}}{g \Delta t^{2}}
$$

where $\Delta p_{x i}$ is the $x$-axis difference between the current and desired ZMP at frame $i, \Delta x_{i}$ is $x$-axis difference between the current and modified $\mathrm{CM}$ at frame $i, z_{c}$ is a constant vertical position of CM, $g$ is a gravity acceleration constant, and $\Delta t$ is a discrete time step. The equation of $y$-axis can be written in a similar way. By solving the linear system consisting of all the frames, we can approximately obtain a $\mathrm{CM}$ trajectory which makes ZMP closer to the desired one, and the accurate solution can be obtained by iterating this calculation.

Equation (9) is derived with the assumption that the vertical $\mathrm{CM}$ position is constant, but actual human motions usually include the vertical movement of CM. To support such motions, we modified equation (9) as follows:

$\Delta p_{x i}=\frac{-z_{i} \Delta x_{i-1}+\left\{2 z_{i}+\left(g+\ddot{z}_{i}\right) \Delta t^{2}\right\} \Delta x_{i}-z_{i} \Delta x_{i+1}}{\left(g+\ddot{z}_{i}\right) \Delta t^{2}}$

where $z_{i}$ is the vertical CM position, which is variable in this equation. We confirmed that this approximation works well for input motions where CM vertically moves, and the system can be more robust for various inputs.

\section{Waist Trajectory Feedback}

In the existing methods, the adjusted waist trajectory is directly applied to the robot model using inverse kinematics between the waist and a support foot. However, it does not consider preserving the spatial relationship between a support leg and the other body parts. Especially in the case where a swing leg or an arm has close interactions with a support leg, the resulting motion could not be similar to the original one and the motion may cause self-collisions.

To solve this problem, the modified waist trajectory is not directly applied to the robot model, but fed back to the IMbased adaptation part as additional hard positional constraint specified to the waist marker. By re-processing the adaptation with the additional constraint, the output is refined so that it can satisfy both the dynamic consistency and the spatial relationships. Then joint angle fitting of the robot model is processed again. Although there may still be some errors between the resulting ZMP and the desired ZMP, the process can be iterated to reduce the error until it is sufficiently small.

\section{EXPERIMENTAL RESULT}

We verified the proposed system using an actual human motion data and applying it to a biped robot model. We implemented the system as a plugin of the Choreonoid robotics software framework [17][18]. We used some motion clips of CMU motion capture database [19] as test motions and the model of biped humanoid robot HRP-4C as a test target robot model. The upper images in Fig.5 shows one of test motion clips. As shown in this figure, there are many close interactions between the arms. 

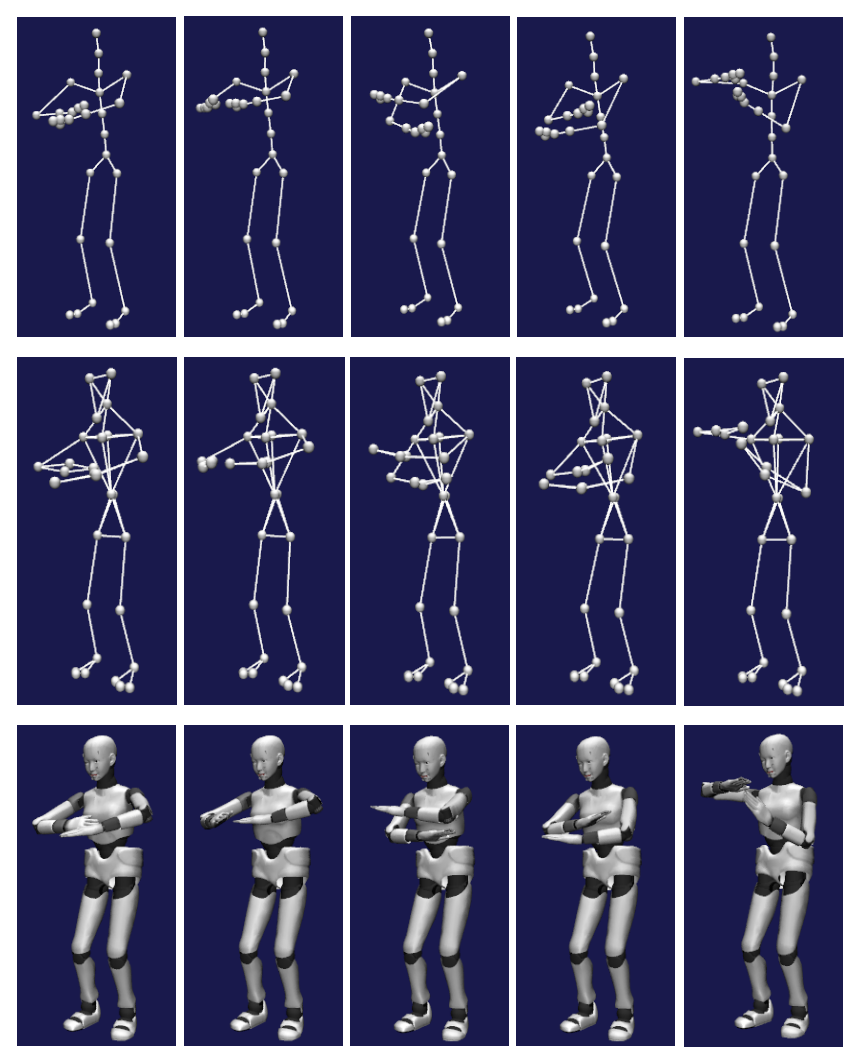

Fig. 5. A sample motion clip and its adapted motions. The upper images show the original motion data. The middle ones show the adapted marker motion represented by the arranged marker model. The lower ones show the final output motion performed by the robot.

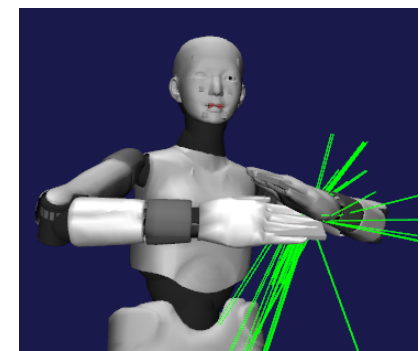

(a)

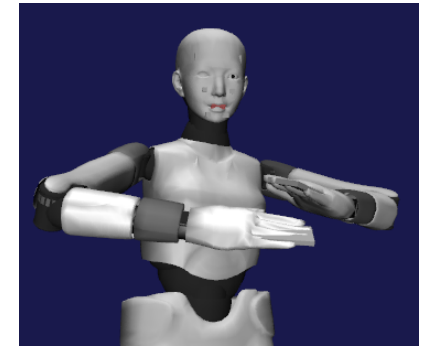

(b)
Fig. 6. A problem caused by direct joint angle fitting. (a) shows the result of the direct fitting for a sample motion. Green lines show collisions between the hands. (b) shows the result with the IM-based adaptation.

When we only applied the marker model arrangement and the joint angle fitting, the resulting motion had self-collisions as shown in Fig.6-(a). Even if collisions does not happen in the original motion, they can be newly produced due to the difference of the kinematic models. On the other hand, when we enabled the IM-based adaptation process, collisions did not happen as shown in Fig.6-(b). The middle images of Fig.5 show the adapted marker motion processed by the marker model arrangement and the IM-based adaptation, and the lower ones show the final motion adapted to the robot joint model. As shown in these images, the spatial relationships between body parts in the adapted motion were sufficiently

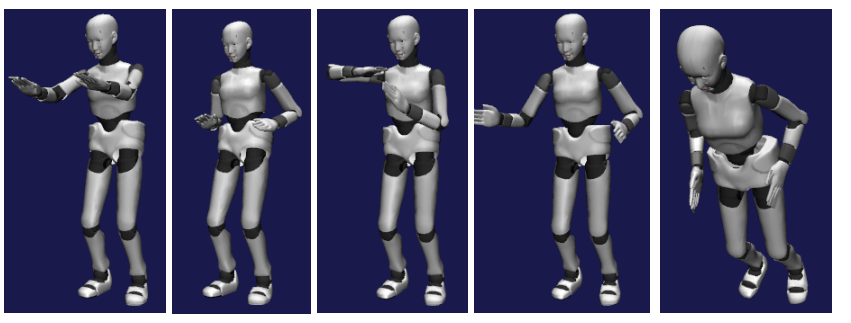

Fig. 7. A result of the dynamics simulation for the adapted motion where the dynamic balance adjustment was not applied.

similar to those of the original motion. The adaptation did not produce any jaggy motions and the trajectories of body parts were as smooth as the original ones.

We also carried out dynamics simulation for the adapted motions. We used a constraint-based dynamics simulator [20] implemented on Choreonoid. When the balance adjustment was not applied, the robot body often swung on the floor and it finally fell down when it performed a large arm movement as shown in Fig.7. A large arm movement can produce large momentum around the contact area if the balance adjustment is not considered well, and it usually results in falling down. On the other hand, the adapted motions with the balance adjustment as shown in the lower images of Fig.5 were stably performed by the HRP-4C model in the dynamics simulation.

\section{CONCLUSION AND FUtURE WORK}

In this paper, we proposed a system which can adapt human motions for a biped humanoid robot. The system can adapt motions while preserving the spatial relationship between body parts and satisfying the dynamic balance consistency for the target robot model. We achieved this system by integrating the marker model arrangement, contact state detection, joint angle fitting, and balance adjustment with the interaction mesh based motion adaptation method. The system allows a given human motion to be smoothly performed by the robot without causing self-collisions and falling down even if the motion includes close interactions between body parts. This is a novel contribution to the problem of adapting human motions for humanoid robots. We confirmed the validity of our system with the experiment using the HRP-4C model and dynamics simulation.

A problem of the current system is that it cannot constrain joint angle ranges and joint angular velocities for a robot model. These factors are also important for moving the actual robot. This problem would be solved by introducing a joint-space morphing process which constrains those factors while considering the deformation energy on the interaction mesh. For that process, the output of the current system would be necessary as an initial input. The system also has a potential to adapt multi-characters motions and motions including close interactions with environmental objects. We will improve the system to be able to support such motions. 


\section{ACKNOWLEDGMENT}

This work was partially funded by the EU FP7 project TOMSY and EPSRC project EP/H012338/1. This work was partially supported by JSPS KAKENHI Grant Number 23240026.

\section{REFERENCES}

[1] S. Nakaoka, K. Miura, M. Morisawa, F. Kanehiro, K. Kaneko, S. Kajita, and K. Yokoi, "Toward the use of humanoid robots as assemblies of content technologies - realization of a biped humanoid robot allowing content creators to produce various expressions "'Synthesiology (English edition), vol. 4, no. 2, pp. 87-98, October 2011, http://www.aist.go.jp/aist_e/research_results/publications/ synthesiology_e/vol4_no2/vol04_02_p87_p98.pdf.

[2] S. Nakaoka, A. Nakazawa, F. Kanehiro, K. Kaneko, M. Morisawa, H. Hirukawa, and K. Ikeuchi, "Learning from observation paradigm: Leg task models for enabling a biped humanoid robot to imitate human dances," International Journal of Robotics Research, vol. 26, no. 8, pp. 829-844, August 2007.

[3] D. Sakamoto, T. Kanda, T. Ono, H. Ishiguro, and N. Hagita, "Android as a telecommunication medium with a human-like presence," in ACM/IEEE international conference on Human-robot interaction. Arlington, Virginia, USA: ACM, March 2007, pp. 193-200.

[4] N. S. Pollard, J. K.Hodgins, M. J. Riley, and C. G. Atkeson, "Adapting human motion for the control of a humanoid robot," in Proceedings of the 2002 IEEE International Conference on Robotics and Automation, Washington DC, April 2002, pp. 1390-1397.

[5] K. Kaneko, F. Kanehiro, S. Kajita, H. Hirukawa, T. Kawasaki, M. Hirata, K. Akachi, and T. Isozumi, "Humanoid robot HRP-2," in Proceedings of the 2004 IEEE International Conference on Robotics and Automation, New Orleans, LA, April 2004, pp. 1083-1090.

[6] K. Yamane, S. O. Anderson, and J. K. Hodgins, "Controlling humanoid robots with human motion data: Experimental validation," in Proceedings of 2010 IEEE-RAS International Conference on Humanoid Robots, Nashville, TN, USA, December 2010, pp. 504-510.

[7] B. Dariush, M. Gienger, A. Arubakkam, Y. Zhu, B. Jian, K. Fujimura, and C. Goerick, "Online transfer of human motion to humanoids," International Journal of Humanoid Robotics, vol. 6, no. 2, pp. 365$289,2009$.

[8] F. Kanehiro, W. Suleiman, K. Miura, M. Morisawa, and E. Yoshida, "Feasible pattern generation method for humanoid robots," in Proceedings of the 9th IEEE-RAS International Conference on Humanoid Robots, Paris, France, December 2009, pp. 542-548.
[9] E. S. Ho, T. Komura, and C.-L. Tai, "Spatial relationship preserving character motion adaptation," in ACM Transactions on Graphics (Proceedings of ACM SIGGRAPH 2010), vol. 29, no. 3. ACM SIGGRAPH, Addison Wesley, 2010.

[10] K. Kaneko, F. Kanehiro, M. Morisawa, K. Miura, S. Nakaoka, and S. Kajita, "Cybernetic Human HRP-4C," in Proceedings of the 10th IEEE-RAS International Conference on Humanoid Robots, Paris, France, December 2009, pp. 7-14.

[11] H. Si and K. Gärtner, "Meshing piecewise linear complexes by constrained delaunay tetrahedralizations," in Proceedings of the 14th International Meshing Roundtable, 2005, pp. 147-163.

[12] M. Alexa, "Differential coordinates for local mesh morphing and deformation," The Visual Computer, no. 19, pp. 105-114, 2003

[13] C. W. Wampler, "Manipulator inverse kinematic solutions based on vector formulations and damped least-squares methods," IEEE Transactions on Systems, Man, and Cybernetics, vol. SMC-16, pp. 93-101, 1986.

[14] Y. Nakamura and H. Hanafusa, "Inverse kinematic solutions with singularity robustness for robot manipulator control," ASME Journal of Dynamic Systems, Measurement and Control, vol. 108, no. 3, pp. $163-171,1986$

[15] M. Vukobratović, A. Frank, and D. Juricic, "On the stability of biped locomotion," IEEE Transaction on Biomedical Engineering, vol. 17, no. 1, pp. 25-36, 1970.

[16] K. Nishiwaki, S. Kagami, Y. Kuniyoshi, M. Inaba, and H. Inoue, "Online generation of humanoid walking motion based on a fast generation method of motion pattern that follows desired ZMP," in Proceedings of the 2002 IEEE/RSJ International Conference on Intelligent Robots and Systems, EPFL, Lausanne, Switzerland, October 2002, pp. 2684-2689.

[17] S. Nakaoka, "Choreonoid as a software framework for implementing graphical robotics applications," in Proceedings of the 29th Annual Conference of the Robotics Society of Japan, Tokyo, Japan, September 2011.

[18] "Choreonoid Official Site," http://choreonoid.org/.

[19] "Carnegie Mellon University Motion Capture Database," http://mocap. cs.cmu.edu/.

[20] S. Nakaoka, S. Hattori, F. Kanehiro, S. Kajita, and H. Hirukawa, "Constraint-based dynamics simulator for humanoid robots with shock absorbing mechanisms," in Proceedings of the 2007 IEEE/RSJ International Conference on Intelligent Robots and Systems, San Diego, CA, USA, October 2007, pp. 3641-3647. 